Volume 2 Issue 1

June 2017

\title{
Influence of Family on Saudi Arabian Emergency Medical Services Students.
}

William J. Leggio EdD NRP

Creighton University, EMS Education, NE, USA

Abdulmajeed Mobrad MD

King Saud University, Saudi Arabia

James R. Martin $\mathrm{PhD}$

Creighton University, NE, USA

Osama Samarkandi PhD RN

King Saud University, Saudi Arabia

Abdullah Mubarak BS

King Saud University, Saudi Arabia

Nabeel Abdulqader BS

King Saud University, Saudi Arabia

Mathew Strehlow MD

Stanford University, CA, USA

\section{Recommended Citation}

Leggio W, Mobrad A, Martin J, Samarkandi O, Mubarak A, Abdulqader N, Strehlow M. Influence of Family on Saudi Arabian Emergency Medical Services Students. 2017 Jun; 2(1).

This is an Open Access article distributed under the terms of the Creative Commons Attribution-Non-Commercial-ShareAlike 4.0 International (http://creativecommons.org/licenses/by-nc-sa/4.0/), which permits use, distribution, and reproduction in any medium, provided the original work and any attributes thereof are properly cited, are distributed under the same licence, and that the work is not used for commercial purposes.

Follow the Irish Journal of Paramedicine online at www.irishparamedicine.com, on Twitter (@irishjparamed) and on Facebook. 
RESEARCH

\title{
Influence of Family on Saudi Arabian Emergency Medical Services Students.
}

\author{
William J. Leggio EdD ${ }^{1}$, Abdulmajeed Mobrad $\mathrm{MD}^{2}$, James R. Martin $\mathrm{PhD}^{2}$, Osama Samarkandi $\mathrm{PhD}^{2}$, Abdullah \\ Mubarak $\mathrm{BS}^{2}$, Nabeel Abdulqader $\mathrm{BS}^{2}$, Mathew Strehlow $\mathrm{MD}^{3}$ \\ 1. Creighton University, EMS Education, School of Pharmacy and Allied Health Professions, NE, USA. 2. King Saud University, Saudi Arabia. 3. \\ Stanford University, CA, USA.
}

Received: 18 Dec $2016 \quad$ Revised: 24 Mar $2017 \quad$ Accepted: 10 May $2017 \quad$ Published: 12 June 2017

Correspondence: Dr. William Leggio, Creighton University, EMS Education. Email: WilliamLeggio@creighton.edu

Abstract

\section{Objectives}

To identify influences on learning for Saudi male students studying Emergency Medical Services at a college in Riyadh, Saudi Arabia. Previous research on influences on student learning in the Kingdom of Saudi Arabia focused on the historical development of education in Saudi Arabia, English language development, and intrinsic motivations of students. Excluded is a focus on students studying Emergency Medical Services.

\section{Methods}

Exploratory sequential mixed-method was applied.

\section{Results}

Family support was an exceptionally strong predictor of student confidence in both skills and post-graduate EMS employment. Concepts involving application, memorization, motivation, and English language did not present as statically significant. The discovery of the strong influences that a family can have on Saudi EMS student's confidence is noteworthy, as this was not previously discovered in the literature.

\section{Conclusions}

This discovery holds practical implications for EMS education and training programs as emphasizes the importance of developing practical ways to include a student's family as a source of support in ensuring student success and confidence.

Keywords: learning barriers; learning influences; emergency medical services; paramedic; Saudi Arabia.

\section{Introduction}

Emergency Medical Services (EMS) bachelor degree programs are emerging in the Kingdom of Saudi Arabia. Graduates of these programs are trained to serve as paramedics and be part of emergency response systems. The training required to achieve these goals is rigorous as hundreds of hours of lecture, practical, and clinical sessions are combined into a program. A literature search on learning barriers while studying Emergency Medical Services (EMS) at a public University in Riyadh, Kingdom of Saudi Arabia was conducted. This search resulted in very limited results. Thus the focus was expanded to learning barriers faced by Saudi Arabian college students. Three strongly supported common learning influences that crossed multi-disciplines were identified as English language, motivation, and the quality within academic institutes starting with the general development of the Saudi education system.

Education in Saudi Arabia developed from two lineages.(1) The first lineage of traditional learning relied on oral communication and was religiously oriented with a curriculum focused on memorizing the Qur'an and the Hadith.(1) The second lineage developed into two types. For many years kuttab instruction was limited to religion, Arabic language, and basic arithmetic. Many modern elementary schools, or madrassa, have developed broader curriculum that retained elements of kuttab instruction, which resulted in the continuation of a preacher-like image of the teacher.(1)

Following considerable international and local pressure, the Ministry of Education implemented the English curriculum at the elementary level beginning in 2003.(1,2 )
Attitudes towards English language education have shifted due to the growing economic, industrial and commercial base in Saudi.(3) The English language classroom in Saudi has been described as complying with the ceremonial order, or the primary role of the teacher and text and the student in the secondary role.(1) Others have described English being taught only for its cultural and intellectual awareness.(2) Observations have been reported on students' difficulty in using the English language for the purpose of studying, reading and examinations, which have resulted in considerable difficulty with academic performance.(4)

A comparative study on motivations for learning the English language of Saudi undergraduates studying medicine, English, and information technology discovered higher levels of extrinsic motivations than intrinsic.(5) The medical students had significantly higher extrinsic motivation than the other populations, which was attributed to practice of only admitting students with the highest grade point average into Saudi medical schools.(5) These findings supported discussions on how English remained as an academic exercise with much greater external pressures to learn than internal.(1-3)

Saudi students hold certain beliefs about learning English that have resulted in poor student attitudes and low levels of motivation, which affects their classroom performance.(3) English is not viewed as immediately relevant to student needs and resulted in students only devoting enough focus to acquire the minimal competency needed to pass a course and not the other aspects of learning 
the language.(3) The lack of intrinsic motivation compounded with the extrinsic influence of the education systems hinders developing any personal interest.(3) Additionally, it has been reported how there are no real purpose or opportunity to use English outside of the classroom.(3)

A review of published literature on the influences on student learning in Saudi Arabia discovered a growing body of research primarily on Saudi students studying English. The review yielded detailed discussions on motivation, barriers to learning English in Saudi Arabia, and generalized findings related to medical students. A gap in identifying the learning barriers faced by EMS students was discovered. This required further research to explore if other influences on student learning found in the literature are the same for bachelor degree seeking Saudi paramedic students.

\section{Methods}

This study addressed influences on learning for students studying EMS at a government college in Riyadh, Kingdom of Saudi Arabia. This study was approved by the ethics board at Prince Sultan bin Abdulaziz College for EMS of King Saud University in Riyadh, Saudi Arabia. The purpose of this exploratory sequential mixed-methods design was to develop and test an instrument to assess learning influences.(6) All participants volunteered to be part of this study. The first phase of the study was a qualitative exploration of learning influences experienced by students studying EMS in Saudi at a public University, for which audio data was collected and analyzed from three focus groups of students. The reason for the qualitative phase was to identify connections between the literature on learning influences on Saudi Arabian students studying English or medicine to EMS students. The quantitative phase followed for the purpose of exploring shared student-learning influences discovered in both the literature review and qualitative findings. The quantitative survey was conducted from March 17, 2015 through April 1, 2015 in Riyadh, Saudi Arabia. A total of 141 out of 143 students participated fully in the quantitative study. The survey was administered using Qualtrics $^{\mathrm{TM}}$, and first written in English then translated to Arabic and agreed upon by three native speakers. Participants were able to read all questions in both English and Arabic.

\section{Study Population}

Study participants, all male undergraduate students in Riyadh, Saudi Arabia, learned EMS from a curriculum comprised of lectures, hands-on skill development sessions, and rotations in both in-hospital departments related to emergency medical care and out-of-hospital emergency services. Lectures and hands-on skill sessions are primarily conducted in the English language. The curriculum is based on international standards, reviewed by local and international EMS experts, and responsive to national need.

\section{Study Design}

In order to explore the influences suggested by the literature, we operationalized several concepts. The purpose of this article was to explore which factors led to students' perceptions of their confidence to serve as EMS practitioners. Thus, factors exploring perceptions of confidence in this preparation, asking about their relative level of confidence in their skills on ambulances, at hospitals, and in obtaining posttraining employment in EMS were analyzed as our dependent variables.

Many potential influences may drive perceptions of confidence. Additional factors explored were, among others, demographics, education, and socio-economic. Operationalized concepts that, after robustness checks, emerged as potentially strong influences that also were statistically sound were presented. These influences include K-12 educational approaches, language skills, the ease of students to participate in the EMS program, the personal motivations of students in pursuing EMS, and finally, the support of family while pursuing EMS studies.

To capture K-12 education and its' impact on students, several facets of primary and secondary education were explored. Two concepts emerged as potentially important to the ability of students to perform the skills necessary in EMS: a tendency to teach through memorization or a tendency to teach skills through application at the high school level. In the quantitative survey, data was captured on the concept of skills training of application and memorization, asking about the perceptions of their high school teaching methods, specifically regarding their focus on teaching through application or through memorization. Note that, despite their seeming conceptual symmetry, response data from these two variables were not correlated.

Language skills of participants with a series of questions about the use of English in high school and with family and friends was explored. Another concept important to our analysis is the relative ease with which a student can attend to their studies in the EMS program. Given that the program involved some online training, one factor that emerged was internet access. As such, questions about student perceptions of the quality and speed of their home internet services were asked. Additionally, participants were also asked how easily students understand the lectures presented within the program.

The concept of personal desire was also an important potential influence. Participants were asked a series of questions, but centered on the desire of participants to serve most directly in EMS within an ambulance service. To capture personal motivations, participants were asked how motivated they were to serve in EMS with an ambulance service.

Finally, to capture the concept of cultural tendencies within Saudi Arabian society to rely heavily on family for life decisions, participants were asked about student perceptions of family support.

Ethical approval for this study was provided by the Research Committee of the Prince Sultan Bin Abdulaziz College for EMS, Kind Saud University (RC-09/14).

\section{Results}

Participants responded to varying levels of family support they enjoy for their EMS studies. The descriptive statistics for the model variables are presented in Table 1 .

To explore the strength of the relationships among our suite of influences and their possible effect on student confidence regarding their skills and post-studies employment prospects, an ordered logistic regression model was used. Three models are presented: one for each dependent variable: Confidence in Employment in EMS, Confidence in Skills: Working on an Ambulance, and Confidence in Skills: Working in a Hospital. From these three models, the effect of significant variables on these dependent variables across the range of the Likert scale response options, then present the marginal effect of Family Support on the three outcomes were examined and presented in Table 2 . 


\begin{tabular}{|l|c|c|c|}
\hline \multicolumn{2}{|l|}{ Table 1. Descriptive Statistics } & Mean & St. Dev. \\
\hline Dependent Variables & 140 & 4.1 & .88 \\
\hline Confidence in Employment in EMS & 140 & 4.2 & .77 \\
\hline Confidence in Skills: Working on an Ambulance & 140 & 3.6 & 1.1 \\
\hline Confidence in Skills: Working in a Hospital & 141 & 3.9 & .91 \\
\hline High School: Memorization Focused & 141 & 3.6 & 1.1 \\
\hline High School: Application Focused & 141 & 2.6 & .95 \\
\hline English Use at High School & 140 & 3.9 & .98 \\
\hline Student Home Internet Quality & 140 & 3.9 & 1.2 \\
\hline Student Motivated to Work in EMS on Ambulance & 140 & 3.7 & .79 \\
\hline Student Understands EMS Lectures & 140 & 4.1 & .94 \\
\hline Family Support for EMS Studies & & & \\
\hline
\end{tabular}

Table 2. Ordered Logistic Regression Models

\begin{tabular}{|lccc|} 
Ordered Logistic Models & $\begin{array}{c}\text { Confidence in Obtaining } \\
\text { Employment in EMS }\end{array}$ & $\begin{array}{c}\text { Confidence in Skills: } \\
\text { Working on an } \\
\text { Ambulance }\end{array}$ & $\begin{array}{c}\text { Confidence in Skills: } \\
\text { Working on an } \\
\text { Ambulance }\end{array}$ \\
\hline High School: Application & -.063 & -.220 & .075 \\
& $(.179)$ & $(.185)$ & $(.171)$ \\
\hline High School: Memorization & .147 & .138 & -.200 \\
& $(.184)$ & $(.206)$ & $.188)$ \\
\hline High School Skills: English Use & .182 & .006 & .291 \\
& $(.186)$ & $(.188)$ & $(.179)$ \\
\hline Internet Quality at Home & $.374^{*}$ & $.341^{*}$ & $.399^{*}$ \\
& $(.171)$ & $(.176)$ & $(.169)$ \\
\hline Motivation: Ambulance Para & .087 & .215 & .094 \\
& $(.145)$ & $(.150)$ & $(.139)$ \\
\hline Family Supports EMS Studies & $.627^{*}$ & .249 & .275 \\
& $(.192)$ & $(.189)$ & $(.181)$ \\
\hline Understands EMS Lectures & $.673^{*}$ & $.707^{*}$ & .184 \\
& $(.231)$ & $(.241)$ & $(.210)$ \\
\hline Log Likelihood & -149.8 & -136.5 & 140 \\
$\mathrm{~N}$ & 140 & 140 & 386.7 \\
\hline AlC & 321.8 & 295.0 & \\
\hline${ }^{*} p$ value $<=.05,2$ sided standard errors in parentheses & & & \\
\hline & & & \\
\hline
\end{tabular}

These models present coefficients rather than odds ratios, allowing for a more conventional interpretation. The results suggested that three of the central influences are related to increases in the confidence of participants in obtaining poststudies employment in EMS as well as their confidence in the skills they need in both ambulance and hospital settings.

These influences are the quality of the participant's home internet, their ability to understand EMS lectures in the school, and the support of family in their studies. The AIC scores, which give a sense of the relative fit of the model when compared to the other models, suggested that the suite of variables included in Model 2 provided the best leverage on perceptions of ambulance skills while Model 3 provided the worst leverage on student perceptions of their skills in a hospital setting. Interestingly, the concepts involving application, memorization, motivation, and English use did not present statistically significant results.

At the same time, given the nature of the Likert scale, a more detailed exploration of these results was needed. Table 3 examines the differentiated response options for each of the significant variables and their impact on the confidence variables.

These results suggest that while Family Support remains consistently significant across the range of responses (from strongly disagree to strongly agree) the results from the other two variables of Student Understands Lectures and Internet Home Quality created a somewhat misleading picture. While those who believe that the quality of their home internet quality was poor seemed to have poor confidence in their skills within a hospital setting, the other response options for this variable are not significant. The range of the response data in this nuanced examination may have washed out what was a significant variable in the main model. As such, focus was placed on Family Support for the remainder of the analysis.

Table 4 displays the marginal effect of Family Support on our three dependent variables.

Figure 1, a marginal effects plot with 95\% confidence intervals around the coefficient estimates, displays the marginal effect of the levels of Family Support on those participants who felt highly confident in their hospital skills. Here, we see that family support appears to have a consistent positive influence on confidence. Any family support at all appears to increase confidence.

Figure 2, another marginal effects plot, examines the effect of increasing levels of family support on the 


\section{Coefficients at Response} Levels

Family Support: Disagree

Family Support: Neutral

\section{Confidence in Obtaining \\ Post-Studies \\ Employment in EMS = \\ Strongly Agree}

\section{Confidence in Skills: Working on an Ambulance $=$ Strongly Agree}

Confidence in Skills: Working in a Hospital = Strongly Agree

\section{Family Support: Agree}

Family Support: Strongly Agree

\begin{tabular}{|lccc|}
\hline Understands Lectures: Neutral & - & -.194 & - \\
Understands Lectures: Agree & - & $(.949)$ & - \\
& - & .616 & - \\
Understands Lectures: Strongly & - & $(.966)$ & - \\
Agree & - & 1.668 & - \\
\hline Internet Home Quality: Disagree & - & $(1.087)$ & $-2.566^{*}$ \\
& - & - & $(1.184)$ \\
Internet Home Quality: Neutral & - & - & -1.491 \\
& - & - & $(1.081)$ \\
Internet Home Quality: Agree & - & - & -.948 \\
& - & - & $(1.005)$ \\
Internet Home Quality: Strongly & - & - & -.476 \\
Agree & - & - & $(1.031)$ \\
\hline * $p$ value $<=.05,2$ sided standard errors in parentheses & & &
\end{tabular}

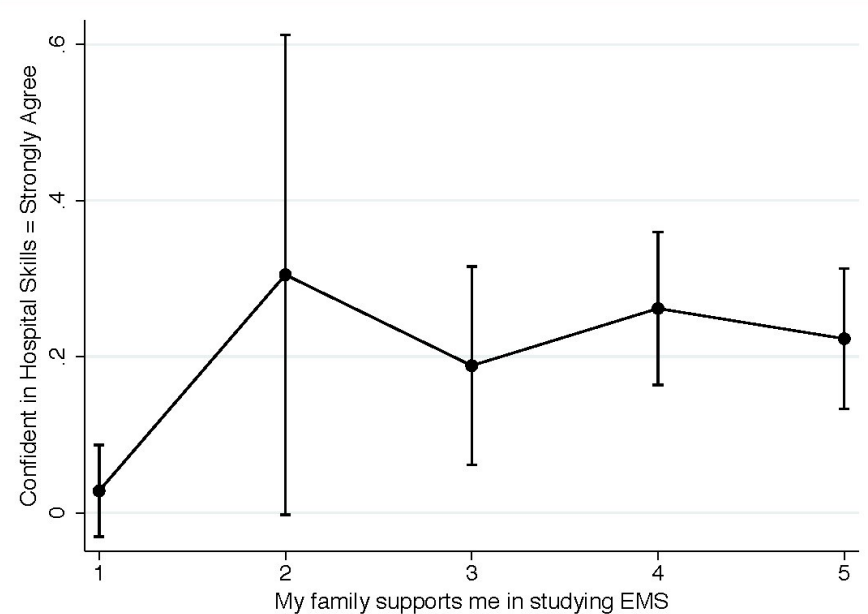

Figure 1. Confidence in hospital skills

perceptions of participants that are highly confident while working in a rescue squad environment. This plot reveals a similar relationship: family support appears to increase EMS student perceptions of confidence in their ambulance skills.

A final marginal effects plot, Figure 3, examines the relationship between various levels of family support on postgraduation employment prospects. This relationship is again

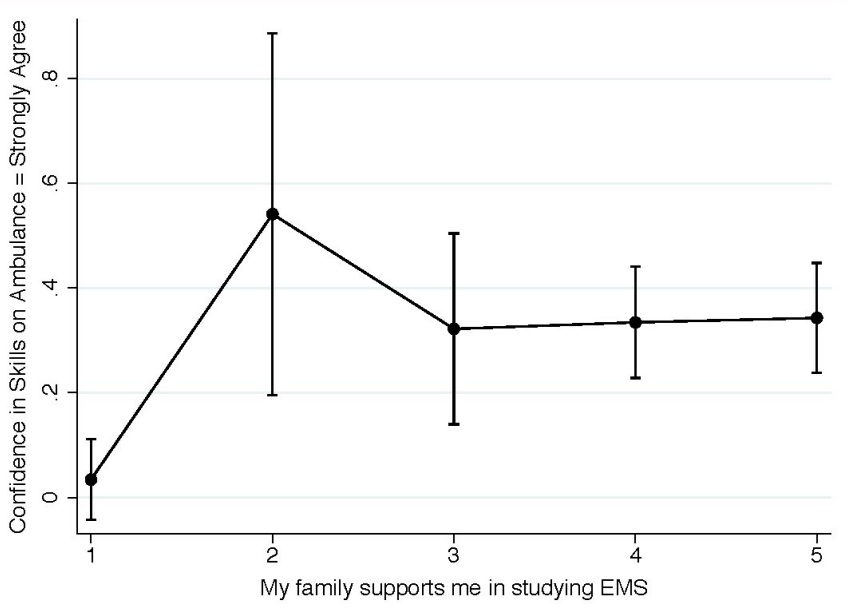

Figure 2. Confidence in ambulance skills

strong and displays a slight upward trend: as student perceptions of the support they receive from their family increased, they also appear to feel more confident about their post-graduation employment prospects in EMS.

\section{Discussion}

The students who felt most confident about their skills 


\section{Marginal Effects: Family Support}

\author{
Confidence in Skills: \\ Working on Ambulance = \\ Strongly Agree
}
Confidence in Skills: Working in Hospital = Strongly Agree

\begin{tabular}{|c|c|c|c|}
\hline \multirow[t]{2}{*}{ Strongly Disagree } & .021 & .034 & .028 \\
\hline & $(.025)$ & $(.039)$ & $(.030)$ \\
\hline \multirow[t]{2}{*}{ Disagree } & $.338 *$ & $.541 *$ & $.305 *$ \\
\hline & $(.160)$ & $(.176)$ & $(.157)$ \\
\hline \multirow[t]{2}{*}{ Neutral } & $.341 *$ & $.322 *$ & $.188 *$ \\
\hline & $(.098)$ & $(.093)$ & $(.065)$ \\
\hline \multirow[t]{2}{*}{ Agree } & .270 * & .334 * & .262 * \\
\hline & $(.049)$ & $(.054)$ & $(.050)$ \\
\hline \multirow[t]{2}{*}{ Strongly Agree } & .490 * & .343 * & .223 * \\
\hline & $(.061)$ & $(.053)$ & $(.046)$ \\
\hline
\end{tabular}

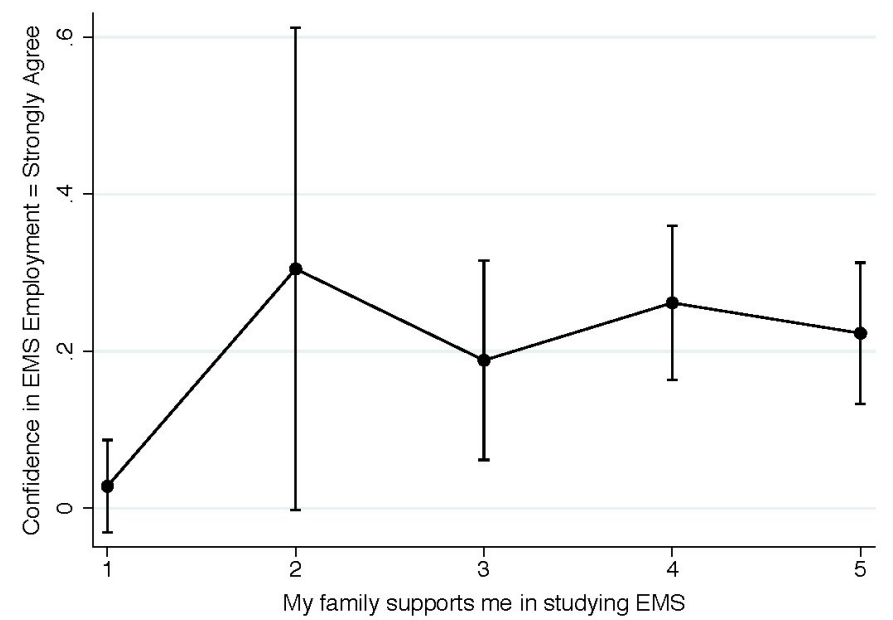

Figure 3. Post-graduation employment prospects

and their post-graduation prospects tended to be those who enjoyed high levels of family support. Figures 1, 2, and 3 support this relationship. This relationship is quite similar in magnitude to the results presented in Figure 1. Those who are highly confident in their skills appear to enjoy strong levels of family support, though in this arena, the relationship increased at roughly the same rate as family support increased. Students who had the most family support expressed the highest confidence in their ability to serve as a paramedic in a hospital or ambulance setting. All the response options presented a statistical significant result except for those students who do not enjoy family support at all.

The results suggested that family support is a strong influence on confidence, whereas concepts involving application, memorization, motivation, and English use were not. These results add to discussions on the role of English use as an academic exercise, and extrinsic influences, such as family support, on learning for Saudi students. However, data and literature on family support being a stronger influence on student learning compared to the other concepts analyzed was not discovered.

The role of family in Saudi Arabia plays a significant role in its culture and is rooted in traditions. The influence of family in EMS student's motivation holds practical implications for education and training centers on discovering ways to incorporate family support as a way to keep students motivated and successful. Thus, further researching the role of family support and expanding how it influences student learning is recommended not only for EMS but other disciplines and specialties as well. Additional investigations on the influence of family on undergraduate, graduate, and professional students would add clarity on this phenomenon as this study only researched undergraduate EMS students.

\section{Limitations}

This study was limited to an all male sample student population studying at an undergraduate EMS college in Riyadh, Saudi Arabia which is part of a government funded public University. An important limitation to note is that these findings are focused on the student's perception of their confidence and not an objective measurement of their skill ability. This study is also limited by the fact that participants completed or nearly completed their elementary education prior to 2003 the Ministry of Education implemented the English curriculum at the elementary level.

\section{Conclusion}

This study examined barriers and influences on male Saudi Arabian students studying EMS in Saudi Arabia. Following a literature review and conducting qualitative focus group interviews, a quantitative survey tool was developed and administered. Within the data, the most important finding was that family support appears to be an exceptional strong predictor of student confidence in both skills and post-graduate EMS employment prospects. The measures of concepts involving application, memorization, motivation, and English did not, for this sample, present statistically significant results. Results from this study help to add to existing literature, but also identified the role of family support for Saudi Arabian students, and perhaps those of other nationalities, studying EMS as a concept requiring further investigation and research.

\section{References}

1. Elyas T, Picard M. Saudi Arabian educational history: Impacts on English language teaching. Education, Business \& Society. 2010;3(2):136-145. 
2. Maherzi S. Perceptions of classroom climate and motivation to study English in Saudi Arabia: Developing a questionnaire to measure perceptions and motivation

Electronic Journal of Research in Educational Psychology. 2011;9(2):765-798.

3. Al-Seghayer $\mathrm{K}$. The four most common constraints affecting English teaching in Saudi Arabia. International Journal of English Linguistics. 2014;4(5):17.

4. Al-Mously N, Salem R, Al-Hamdan N. The impact of gender and english language on the academic performance of students: An experience from new Saudi medical school. Journal of Contemporary Medical Education. 2013;1(3):170176.

5. Javid CZ, Al-Asmari A, Farooq U. Saudi undergraduates' motivational orientations towards English language learning along gender and university major lines: A comparative study. European Journal of Social Sciences. 2012;27(2):283300.

6. Creswell JW, Plano Clark VL. Designing and conducting mixed methods research. Thousand Oaks, California: Sage Publications; 2007.

Author contributions: All authors contributed to the writing and final editing of the manuscript.

Source of support/funding: None.

Conflict of interest: WJL is Associate Editor of the IJP and a member of the IJP Editorial Board. No other conflicts of interest were declared.

Provenance and review: Not commissioned, peerreviewed.

This is an Open Access article distributed under the terms of the Creative Commons Attribution-Non-Commercial-ShareAlike 4.0 International (http:// creativecommons.org/licenses/by-nc-sa/4.0/), which permits use, distribution, and reproduction in any medium, provided the original work and any attributes thereof are properly cited, are distributed under the same licence, and that the work is not used for commercial purposes. Content copyright remains with the authors, who grant the IJP a licence to reuse and distribute. 